\title{
Earlier autism diagnosis associated with greater trait improvement
}

\section{BY PETER HESS}

2 DECEMBER 2021

Children who are diagnosed with autism before 2 and a half years of age show greater gains in their social skills, on average, than children who are diagnosed later, according to a new study.

Early diagnosis is widely thought to be important because it makes it possible to treat children early, yet there has been little evidence that early treatment benefits autistic children, says lead investigator Ilan Dinstein, associate professor of cognitive and brain sciences at Ben-Gurion University of the Negev in Beersheba, Israel.

Autistic children who receive early intervention as part of a clinical trial often display promising gains, but those treated in community settings do not typically experience as many benefits, previous studies show.

This gap raised a burning question for Dinstein and his colleagues: "Is there a reason to hurry and perform the diagnosis early in situations where intervention is messy, heterogeneous and not delivered by world-renowned experts?" he says.

The answer, according to the results Dinstein and his colleagues published in October in Autism, appears to be yes.

"I hope that these and related findings will put continued pressure on advisory bodies that produce guidelines, and also on insurers to push for children to be screened for autism earlier in life, and for healthcare professionals to act quickly in the presence of a positive screen," says David Mandell, professor of psychiatry at the University of Pennsylvania in Philadelphia, who was not involved in the study. Mandell is the outgoing editor-in-chief of Autism but did not edit this particular study.

\section{Social-communication boost:}




\section{Spectrum | Autism Research News}

https://www.spectrumnews.org

Dinstein and his team gathered data on 131 children diagnosed with autism at a clinic in Israel; 57 were diagnosed before they were 2 and a half years old, and 74 were diagnosed after — at an average age of 3.1 years. At the time of diagnosis and again one to two years later, clinicians administered the Autism Diagnostic Observation Schedule (ADOS) to measure autism traits and asked parents how many hours per week their children received interventions of any type.

About 65 percent of the children diagnosed early showed significant improvement on the social affect domain of the ADOS during the 1 to 2 years following their diagnosis. By contrast, about 23 percent of the children diagnosed later showed significant improvement during the same period.

The children diagnosed earlier who showed improvements on the social affect domain of the ADOS also showed greater improvements on this measure than the later-diagnosed children. The two groups in the study received similar numbers of hours of interventions, and the type of intervention had no significant effect on outcomes.

The children who were diagnosed earlier tended to have more severe social affect scores at diagnosis and less severe restricted and repetitive behavior ( $R R B$ ) scores than the laterdiagnosis group. But neither group showed a statistically significant change over time in RRBs. The finding suggests that early interventions, which disproportionately focus on social communication, should also focus on RRBs, Dinstein says.

A comparison of early- and late-diagnosed children who started out with comparable ADOS scores again revealed that about three times as many early-diagnosed children show significant improvements on the ADOS as late-diagnosed children - confirming that the findings do not just reflect differences in trait severity at the time of diagnosis.

"I think it's the first time a direct comparison was performed between those diagnosed before 2 and a half years of age and those diagnosed later," Dinstein says. "The difference in social communication outcome is immense. I didn't think it would be so large."

\section{Results may vary:}

Dinstein and his team chose 2 and a half years as a cutoff because "most autism screening tools are designed to identify autism between the ages of 1 and a half and 2 and a half years," he says.

This cutoff also captures a unique time window in children's relationships with clinicians, says Pamela DeGuzman, associate professor of nursing at the University of Virginia in Charlottesville, who was not involved in the study. After about 18 months of age but before 4 years, once a baby's immunizations are completed, parents tend to feel less urgency about bringing their child to the doctor. "They know vaccinations are a thing, but they're not as tuned in to screening for developmental delays." 


\section{Spectrum | Autism Research News}

https://www.spectrumnews.org

But these routine doctor visits represent crucial opportunities for health-care providers to emphasize how important early autism screening is, she says. "We now have even more evidence to show that it's really important to get kids screened during their well-child visits."

The findings also underscore the importance of referring children for early intervention, Mandell says. "We often put emphasis on the screening and diagnosis, and we don't necessarily put as much emphasis on making sure that those kids are quickly triaged to appropriate care, as if there was something magical about an earlier diagnosis."

The current study focused only on core autism traits, not cognitive outcomes or adaptive behaviors. Dinstein and his group are following another participant group to assess the degree to which age at diagnosis and intervention type and intensity affect these factors.

Cite this article: https://doi.org/10.53053/FYGC3433 